\title{
A Novel Approach to the Design and Analysis of Field Experiments to Study Variation in the Tolerance and Resistance of Cultivars to Root Lesion Nematodes (Pratylenchus spp.)
}

\author{
Karyn L. Reeves, ${ }^{1,2, \dagger}$ Clayton R. Forknall, ${ }^{3}$ Alison M. Kelly, ${ }^{3}$ Kirsty J. Owen, ${ }^{4}$ Joshua Fanning, ${ }^{5}$ Grant J. Hollaway, ${ }^{5}$ and \\ Robert Loughman ${ }^{1}$ \\ ${ }^{1}$ Department of Primary Industries and Regional Development, South Perth, Western Australia, 6151, Australia \\ ${ }^{2}$ Statistics for the Australian Grains Industry (SAGI) West, Curtin University, Bentley, Western Australia, 6102, Australia \\ ${ }^{3}$ Department of Agriculture and Fisheries, Leslie Research Facility, Toowoomba, Queensland, 4350, Australia \\ ${ }^{4}$ Centre for Crop Health, University of Southern Queensland, Toowoomba, Queensland, 4350, Australia \\ 5 Agriculture Victoria, Horsham, Victoria, 3400, Australia \\ Accepted for publication 27 May 2020.
}

\begin{abstract}
The root lesion nematode (RLN) species Pratylenchus thornei and $P$. neglectus are widely distributed within cropping regions of Australia and have been shown to limit grain production. Field experiments conducted to compare the performance of cultivars in the presence of RLNs investigate management options for growers by identifying cultivars with resistance, by limiting nematode reproduction, and tolerance, by yielding well in the presence of nematodes. A novel experimental design approach for RLN experiments is proposed where the observed RLN density, measured prior to sowing, is used to condition the randomization of cultivars to field plots. This approach ensured that

and resistance was undertaken in a linear mixed model framework. Yield response curves were derived using a random regression approach and curves modeling change in RLN densities between sowing and harvest were derived using splines to account for nonlinearity. Groups of cultivars sharing similar resistance levels could be identified. A comparison of slopes of yield response curves of cultivars belonging to the same resistance class identified differing tolerance levels for cultivars with equivalent exposures to both presowing and postharvest RLN densities. As such, the proposed design and analysis approach allowed tolerance to be assessed independently of resistance.
\end{abstract} all cultivars were exposed to consistent ranges of RLN in order to derive valid assessments of relative cultivar tolerance and resistance. Using data from a field experiment designed using the conditioned randomization approach and conducted in Formartin, Australia, the analysis of tolerance
Keywords: disease control and pest management, experimental design, nematology, population biology, random regression analysis, resistance, tolerance
Root lesion nematodes (RLNs) are microscopic plant parasites that feed and reproduce inside the roots of susceptible plants causing loss of root function and symptoms of nutrient and moisture deficiency (Thompson et al. 2012; Whish et al. 2014), which can result in reduced yields. Pratylenchus thornei and P. neglectus are widespread within cropping regions of Australia and therefore have the potential to limit grain production (Vanstone et al. 2008). As RLNs are polycyclic, high densities of RLN can build-up during the growing season in conducive environmental conditions when susceptible crops are grown. This has the potential to reduce the yields of intolerant crops grown not only in the current season but also in subsequent seasons. Annual combined losses in production for the wheat (Triticum aestivum) industry, due to RLN infestation across Australia, have been estimated at A \$123 million (Murray and Brennan 2009).

Cultivars vary in their ability to suppress RLN reproduction and can be ranked along a scale from susceptible to resistant (Fanning et al. 2018; Roberts 2002; Trudgill 1991). In addition, cultivars vary with respect to the impact of RLNs upon grain yield and can be

†Corresponding author: K. Reeves; karyn.reeves@agric.wa.gov.au

Funding: Financial support provided by Grains Research and Development Corporation grants DAW00245 and DAV00128.

*The $e$-Xtra logo stands for "electronic extra" and indicates one supplementary figure is published online.

The author(s) declare no conflict of interest.

(c) 2020 The American Phytopathological Society ranked along a scale from intolerant to tolerant (Roberts 2002; Trudgill 1991). Cultivar resistance and tolerance are believed to be under separate selection pressure and genetic control (Kause and Odegard 2012; Råberg et al. 2009; Sheedy and Thompson 2009). The requirement to remain profitable impels farmers to select cultivars using a number of criteria, with yield potential and grain quality typically being the primary features, followed by response to constraints such as RLNs. An immediate economic approach may be to select tolerant cultivars that lose less yield compared with other cultivars, while withstanding an equivalent RLN population density. A more effective and sustainable approach would be to select resistant cultivars that do not increase, and can potentially decrease, nematode densities, thereby minimizing yield losses in current and subsequent seasons (Fanning et al. 2020; Owen et al. 2014).

While much of the research on ranking cultivars for resistance is done in glasshouses where manipulation and control of RLNs is facilitated through inoculation and is therefore easier to achieve than in the field (Sheedy et al. 2015), the results cannot be used to relate RLN densities to disease intensity or yield under field conditions (Barker and Olthof 1976). For this reason, RLN experiments for tolerance are conducted in the field and this brings the issue of how RLN densities should be manipulated to ensure all cultivars are exposed to comparable RLN density ranges. Early approaches sought to induce two levels of high and low nematode population densities, with nematicides used as a treatment in the low population plots (Kagoda et al. 2011; Reen et al. 2014; Smiley et al. 2005; Taylor et al. 1999; Thompson et al. 2012), or as field experiments using naturally occurring RLN levels (Taylor et al. 1999; Vanstone et al. 1998). Nicol et al. (1999) described an 
alternative differential cropping approach based on a method used for white potato cyst nematode (Globodera pallida) (Elston et al. 1991). The experiment reported by Nicol et al. (1999) was conducted over two-years, with the first year operating as a set-up year with a range of RLN densities induced through the use of cultivars comprising barley (Hordeum vulgare), oat (Avena sativa), durum wheat (Triticum turgidum ssp. durum), bread wheat (T. aestivum), canola (Brassica napus), and linseed (Linum usitatissimum) cultivars and the incorporation of adjacent fallow plots. The second year operated as an experimental year focused on the cultivars of interest. A similar approach has been used in experiments described in Owen et al. (2014), Reen et al. (2014), and Fanning et al. (2020).

It is a fundamental principle of experimental design that treatments should be allocated to plots using a method of chance, and an element of good experimental practice that plots should be as uniform as possible to avoid confounding with other sources of variability (Fisher 1935). These practices underpin the inference that any differences observed are due to the treatments, and allow statements of cause and effect to be derived. The use of different crops to establish an experiment may affect soil structure, soil water and/or nutrient levels, and the densities of other soilborne pathogens and beneficial organisms such as mycorrhizal fungi (Barker and Olthof 1976) thus potentially confounding the experiment in the second year, even where cultivars are randomly allocated to plots. Furthermore, the practice of incorporating adjacent fallow plots into the experiment nonrandomly, as described in Nicol et al. (1999), has the potential to introduce confounding with an unobservable covariate into the experiment. The use of nematicides does not offer a viable alternative solution to the method of manipulating within-plot RLN densities using set-up cultivars, as they can stimulate plant growth in the absence of pests (Barker et al. 1988), result in increased nutrient availability (Nicol et al. 1999; Thompson et al. 2012), provide poor control of nematodes below $30 \mathrm{~cm}$ soil depth (Reen et al. 2014) and/or cause suppression of other soilborne pathogens and beneficial mycorrhizal fungi (Reen et al. 2014) compared with untreated plots. Therefore, it may be invalid and potentially erroneous to relate nematode density to yield loss when nematicides are used. An analogous argument can be made where bare-fallow treatments are used to establish low densities in paired plots.

Recently, tolerance of wheat and barley cultivars were inferred from grain yield of cultivars sown into field plots infested with low and high RLN densities set up using the cultivar approach (Linsell et al. 2015). However, establishment of only two RLN density levels does not provide sufficient information to fit curves that describe the rate of change in yield in response to increasing nematode population density (i.e., tolerance as defined in Forknall et al. 2019; Råberg et al. 2009) as there is no basis for assessing conformity with the inherent assumption of a linear relationship between the dependent and independent variables. Furthermore, outlying observations have the potential to strongly influence the fit (Gomez and Gomez 1984, page 422). Randomizing cultivars of interest across naturally occurring infestations may avoid the problems in curve-fitting from paired low/high plots but does not ensure that all cultivars are exposed to equivalent ranges of RLN pressure within an environment. An improvement on these experimental design approaches involves establishing a continuous range of nematode densities using three or more cultivars with varying resistance/susceptibility to the target nematode species during the set-up year, as described by Fanning et al. (2020). A limitation of the approach by Fanning et al. (2020), however, is that randomization of the second year cultivars to treatments is based on assumed RLN density levels associated with the set-up cultivars rather than the actual presowing RLN densities measured in each plot. Given the variability of nematode multiplication in the field, randomization of cultivars based on an assumed RLN density level may bias the result with respect to one or more cultivars by chance.
An experimental design approach that results in a wide range of RLN densities and simultaneously controls the range of disease exposure for each cultivar is necessary to ensure the valid assessment and comparison of tolerance and resistance across cultivars. We describe a novel experimental design approach where the randomization of cultivars of interest is conditioned on the RLN plot densities measured before sowing. While the conventional approach to blocking in a field experiment forms blocks from contiguous experimental units to adjust for variation between experimental units, blocks were formed in our approach from groupings of the measured RLN density distribution before sowing $(P i)$ in the experimental year (Kelly and Forknall 2020). This approach adapts a technique used in animal and clinical experiments whereby blocking in the assignment of treatments to experimental units is imposed on the basis of an observed quantity of a continuous variable. In experiments involving animals, for example, weight ranges can be used to define blocks, while in clinical trials, disease severity can be used to block patients (Bailey 2008). A difference in our application is that while treatments were allocated across these RLN blocks, $P i$ formed a treatment of interest in the experiment and so was considered as a treatment rather than a blocking term in the analysis. Where cultivars are exposed to consistent ranges of RLN densities and where the relationship is linear (or can be transformed to linear), tolerance will be described by the slope of a regression line fitted between a measure of yield and a measure of RLN population density (Forknall et al. 2019; Råberg et al. 2009). Differences in cultivar tolerance can be determined through testing the hypothesis of parallel slopes across cultivars (Forknall et al. 2019; Råberg et al. 2009).

The aims of this study were to (i) propose an alternative experimental design approach that conditions the randomization on the observed plot densities to ensure all cultivars are exposed to similar ranges of RLN densities at sowing while controlling for differences in uniformity introduced through differential cropping set-up approaches, and (ii) illustrate the analysis of tolerance and resistance data in a single experiment using a random regression approach to derive curves modeling the yield response and incorporating splines to derive curves modeling the change in nematode densities. Field experiments conducted in Longerenong, Victoria during 2013 and 2014 and Formartin, Queensland during 2014 and 2015 provided motivating data sets to explore the limitations of the conventional approach and the application of the proposed design and analysis methodology, respectively.

\section{MATERIALS AND METHODS}

Motivating example 1 (Longerenong). A 2-year experiment at Longerenong, Victoria, Australia $\left(36.6729^{\circ} \mathrm{S}, 142.2993^{\circ} \mathrm{E}\right.$; 144 m elevation) in 2013 and 2014 was conducted to compare yield performance in the presence of $P$. neglectus among a set of five cereal cultivars ranging from moderately resistant to susceptible to P. neglectus (https://www.grdc-nvt.com.au/home). The experiment was located in a paddock managed to facilitate RLN research. Pathogen population densities were monitored regularly, prior to the initialization of the field experiment, to ensure the target nematode was the only pathogen present (Fanning et al. 2018). The soil at the experimental location is a Black Vertosol (Isbell 1996). Annual rainfall was $361 \mathrm{~mm}$ in 2013 and $267 \mathrm{~mm}$ in 2014.

The experiment was laid out in a rectangular grid of six columns and 15 rows comprising 90 plots with dimensions of $8 \times 1.4 \mathrm{~m}$. In the first year of the experiment, the triticale cultivar Tahara $(\times$ Triticosecale spp.), durum cultivar Tamaroi, and wheat cultivar Young were sown in sets of five contiguous plots to form main plots laid out according to a row-column design, with the intention of establishing low, medium and high $P$. neglectus densities, respectively. In the second year of the experiment, wheat cultivars Impose CL Plus, Merlin, Lincoln, and Estoc and barley cultivar Skipper were randomized to plots within each set-up cultivar main plot 
(Supplementary Fig. S1). Soil samples were taken from each plot 35 days before sowing in the second year. Each sample consisted of 20 cores (150 $\mathrm{mm}$ deep and $15 \mathrm{~mm}$ diameter), taken evenly across the plot using an Accucore sampler (Spurr Soil Probes, Adelaide, Australia). The cores from each plot were pooled to provide a 300 to $500 \mathrm{~g}$ soil sample that was submitted for analysis by PREDICTA B (Ophel-Keller et al. 2008) at the South Australian Research and Development Institute (Adelaide, Australia) to quantify $P$. neglectus densities $(P i)$ using quantitative polymerase chain reaction (qPCR). DNA standards were used to convert the qPCR results to the population density of $P$. neglectus (number per gram of dried soil) to estimate initial $P$. neglectus densities per plot. PREDICTA B has previously been shown to provide estimates of nematode population densities that are highly correlated with other methods of nematode extraction, identification, and enumeration (Hollaway et al. 2003; Owen et al. 2010, 2014). The experimental design for the set-up and experimental years with plot $P i$ estimates are provided in Supplementary Figure S1.

Motivating example 2 (Formartin). A field experiment was conducted in 2014 and 2015 near Formartin, Queensland, Australia $\left(27.4640^{\circ} \mathrm{S} 151.4262^{\circ} \mathrm{E} ; 364 \mathrm{~m}\right.$ elevation) to assess the yield loss of wheat cultivars due to $P$. thornei. The experiment was conducted on a site that has been managed for research purposes for more than 20 years and P. thornei is the only RLN present (Thompson et al. 1999). The soil is a Black Vertosol (Isbell 1996). Annual rainfall was $564 \mathrm{~mm}$ in 2014 and $648 \mathrm{~mm}$ in 2015. The experiment was laid out in a rectangular grid of four columns and 24 rows comprising 96 plots with dimensions of $8 \times 1.75 \mathrm{~m}$. The first year of the experiment operated as a set-up year with a set of wheat cultivars and lines with different resistance ratings to P. thornei (EGA Gregory, Kennedy, QT8343, and Strzelecki) allocated to plots at random using a randomized complete block design to induce a range of $P$. thornei densities across the experiment. Six plots within each block of six rows by four columns were randomly allocated to each set-up cultivar. The second year of the experiment operated as an experimental year with six commercial Australian bread wheat cultivars known to vary in their response to P. thornei (EGA Gregory, Lang, Lincoln, Spitfire, Strzelecki, and Suntop) allocated to plots using a $P i$-conditioned randomization approach described in the following section.

Soil samples were collected from each plot 8 weeks prior to sowing and 3 weeks postharvest for estimation of the initial $(P i)$ and final $(P f) P$. thornei densities by the PREDICTA B testing service. The $P i$ samples comprised two cores taken from the middle rows using a 43-mm-diameter foot-stomper corer (Thompson et al. 1989), while the postharvest samples comprised eight cores taken in zig-zag formation using a small corer $(19.05 \times 1.2 \mathrm{~mm}, 300 \mathrm{~mm}$ long, Rimik, Toowoomba, Australia). Yields were determined through machine harvest at plant maturity on 11 November 2015, with a harvest weight recorded for each plot. Plot weights were corrected for $12 \%$ moisture and converted to tonnes per hectare for data analysis.

Initial density $(\mathrm{Pi}$ )-conditioned randomization experimental design. The conditioned randomization design approach was devised for implementation using a model-based design framework and requires the definition of six model terms: (i) setupVar, a factor with $n_{\mathrm{S}}$ levels describing the set of cultivars used in the set-up year, (ii) expVar, a factor with $n_{\mathrm{E}}$ levels describing the set of cultivars used in the experimental year, (iii) setupBlock, a factor with $r_{\mathrm{S}}$ levels describing replicate blocks of the set of set-up cultivars, (iv) RLNBlock, whose derivation is described below, (v) Row, denoting the rows of the experimental layout, and (vi) Column, denoting the columns of the experimental layout. The number of plots in the field experiment is given by $p_{\mathrm{E}}=r_{\mathrm{S}} \times n_{\mathrm{S}} \times n_{\mathrm{E}}$. The conditioning on $P i$ is implemented through blocks formed using groups of the observed presowing RLN density distribution $(P i)$ in the experimental year, to create the factor RLNBlock with $N$ $=p_{\mathrm{E}} / n_{\mathrm{E}}$ ordinal levels. Plots are assigned to each level by partitioning the $P i$ observations into $N$ groups, with plots with the lowest $n_{\mathrm{E}} P i$ observations assigned to the first level, and plots with the next lowest $n_{\mathrm{E}} P i$ observations assigned to the second level, and so on. RLNBlock is therefore a rank transformation of the variable $P i$ with ranks grouped in blocks of equal size, and is included in place of $P i$ in the model used to generate the design.

A model-based design approach relies on an underlying variance model, together with an optimality criterion, to permute levels of a nominated treatment factor in the search for an optimal design. All of the terms defined above are fit as random effects in the modelbased design framework and, as such, contribute to the variance model. Control over the relative contribution of each term to the variance model is achieved through the specification of variance component estimates. Relatively large variance component estimates are assigned for the RLNBlock and setupVar factors in order to encourage a binary or balanced allocation of experimental cultivars with respect to these terms, such that each experimental cultivar appears in each RLNBlock level exactly once and across the set-up cultivars an approximately equal number of times. Column and Row terms are specified with moderate variance components to balance the allocation of experimental cultivars across these spatial factors, mitigating the potential bias induced from management practices aligned with these field dimensions.

The $P i$-conditioned randomization design approach was used to design the experimental year of the Formartin experiment. It was implemented using the model-based design package, od (Butler 2013), in the R statistical computing software environment (R Core Team 2019). The permute and swap options of the od() function were used to permute the experimental cultivars (expVar) within the original replicate blocks used in the set-up year by specifying setupBlock as the swap term. The randomization was inspected to ensure that all constraints were met, with some flexibility permitted in the balancing of experimental cultivars across set-up cultivars to ensure a solution was feasible. An example call to the od() function is given below:

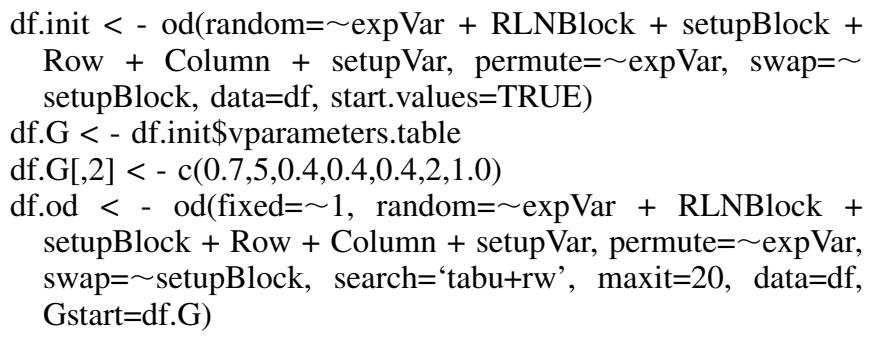

Simulations. The $P i$-conditioned randomization approach was applied to the $P i$ data and plot structure of the Longerenong experiment using a simulation approach to compare the distributions of $P i$ to which each experimental cultivar was exposed under the $P i$-conditioned randomization approach. One hundred randomizations were considered adequate for demonstration purposes. The randomizations were summarized by calculating the interquartile range (IQR) of $P i$ for each cultivar, where the IQR gives the difference between the first and third quartiles and is a robust measure of dispersion for skewed data.

Modeling the yield response. In order to describe the yield response to nematode infestation, curves were fitted to model the relationship between grain yield and $P$. thornei population density, with separate curves fitted for each cultivar of interest so that relative cultivar tolerance could be compared. The random regression approach for deriving response curves in a linear mixed model framework described by Forknall et al. (2019) was used to estimate response curves for the experiment, with separate analyses conducted for the $P i$ and $P f$ data.

On the basis of an assumption that estimates of plot $P i$ and $P f$ data taken from an experiment are approximately log-normally distributed, the $P i$ and $P f$ data were natural $\log$ transformed prior to 
fitting response curves to linearize the relationships between the explanatory variables and the yield observations. The natural log transformation also reduces the potential excess leverage exerted by large observed $P i$ and $P f$ values. This application of the method described in Forknall et al. (2019) models the relationship between yield and the log transformed $P$. thorne $i$ values in the experiment as fixed effects while simultaneously modeling cultivar-specific variation as random deviations around the overall yield profile. A term was included to model covariance between random intercept and slope terms, and additional terms can be included to model local and global spatial trend and small-scale variation (Gilmour et al. 1997; Stefanova et al. 2009). Graphical summaries of the residual distributions were assessed visually to verify model assumptions. Estimates of the overall linear response were derived from the model as empirical best linear unbiased estimators (e-BLUEs), while the estimates of the cultivar-specific random intercept and slope effects were derived as empirical best linear unbiased predictors (e-BLUPs). The e-BLUEs and e-BLUPs were combined to derive intercept and slope values, with the slope values of the respective analyses providing estimates of the cultivar-specific rates of change in yield in response to unit increase in log transformed $P i$ and $\log$ transformed $P f$. The null hypothesis of zero slope was tested for each cultivar by constructing $95 \%$ confidence intervals for each slope estimate. Where appropriate, posthoc comparisons of slope estimates for pairs of cultivars were conducted by testing the null hypothesis of equal slopes and using a Bonferroni correction to accommodate multiple tests. Technical constraints on inducing zero RLN levels under field conditions mean that intercept values, especially those derived from a model fitted to log transformed data, cannot be interpreted as cultivar yield potentials, as extrapolating beyond the range of observed data, and making assumptions about whether such a relationship would be linear, is inappropriate. By controlling the range of $P i$ values to which each cultivar was exposed through the experimental design, these relative rates of change of yield were calculated over an approximately consistent range of $P i$ values.

Modeling the change in nematode density. Curves were fitted to model the change in P. thornei densities between sowing and harvest, with separate curves fitted for each cultivar. As the curves show how the rate of RLN replication across the growing season for an individual experiment varies according to the presowing density for each cultivar, they allow an assessment of relative cultivar resistance to be derived. Spline terms were incorporated in a random regression linear mixed model approach to model nonlinearity in the relationship between $P i$ and $P f$. This means that no assumption of functional form was made with respect to the relationship between initial and final $P$. thornei density for each cultivar, and the observed data were used to inform the underlying relationships.

The response variable of square root transformed $P f$ was modeled with a fixed effect for untransformed $P i$, and with cultivar-specific variation and curvature modeled as random deviations around the overall profile. The $P f$ data were square-root transformed to stabilize the variance, with the transformation selected heuristically following inspection of the residual distribution. The spline approach implemented in ASReml-R (Butler et al. 2018) fits a cubic smoothing spline with one knot for each observation and allows the simultaneous estimation of fixed and random effects and variance modeling (Verbyla et al. 1999), with penalized regression coefficients estimated as random effects and optimal smoothing parameters estimated by residual maximum likelihood (REML) as

TABLE 1. A comparison of the interquartile ranges (IQR) of Pi (Pratylenchus sp. initial population density measured prior to sowing) distributions of $P$. neglectus/g of soil to which each cultivar was assigned under the original randomization approach and under the constrained randomization approach in the Longerenong experiment

\begin{tabular}{lccccc}
\hline & \multicolumn{5}{c}{ Experimental design type } \\
\cline { 2 - 6 } & $\begin{array}{c}\text { Original } \\
(n=1)\end{array}$ & \multicolumn{4}{c}{$\begin{array}{c}\text { Simulated constrained } \\
\text { randomizations }(n=100)\end{array}$} \\
\cline { 2 - 6 } \cline { 5 - 7 } Cultivar & $\begin{array}{c}\text { Observed } \\
\text { IQR }\end{array}$ & $\begin{array}{c}\text { Minimum } \\
\text { IQR }\end{array}$ & $\begin{array}{c}\text { Mean } \\
\text { IQR }\end{array}$ & $\begin{array}{c}\text { Maximum } \\
\text { IQR }\end{array}$ & $\begin{array}{c}\text { Standard } \\
\text { error } \\
(\mathrm{IQR})\end{array}$ \\
\hline Estoc & 51.5 & 18.9 & 23.5 & 25.9 & 0.14 \\
Impose CL Plus & 20.8 & 20.1 & 23.1 & 26.1 & 0.12 \\
Lincoln & 21.0 & 19.2 & 22.7 & 26.2 & 0.14 \\
Merlin & 19.3 & 17.1 & 23.3 & 25.6 & 0.15 \\
Skipper & 22.5 & 19.4 & 22.8 & 26.7 & 0.14 \\
\hline
\end{tabular}
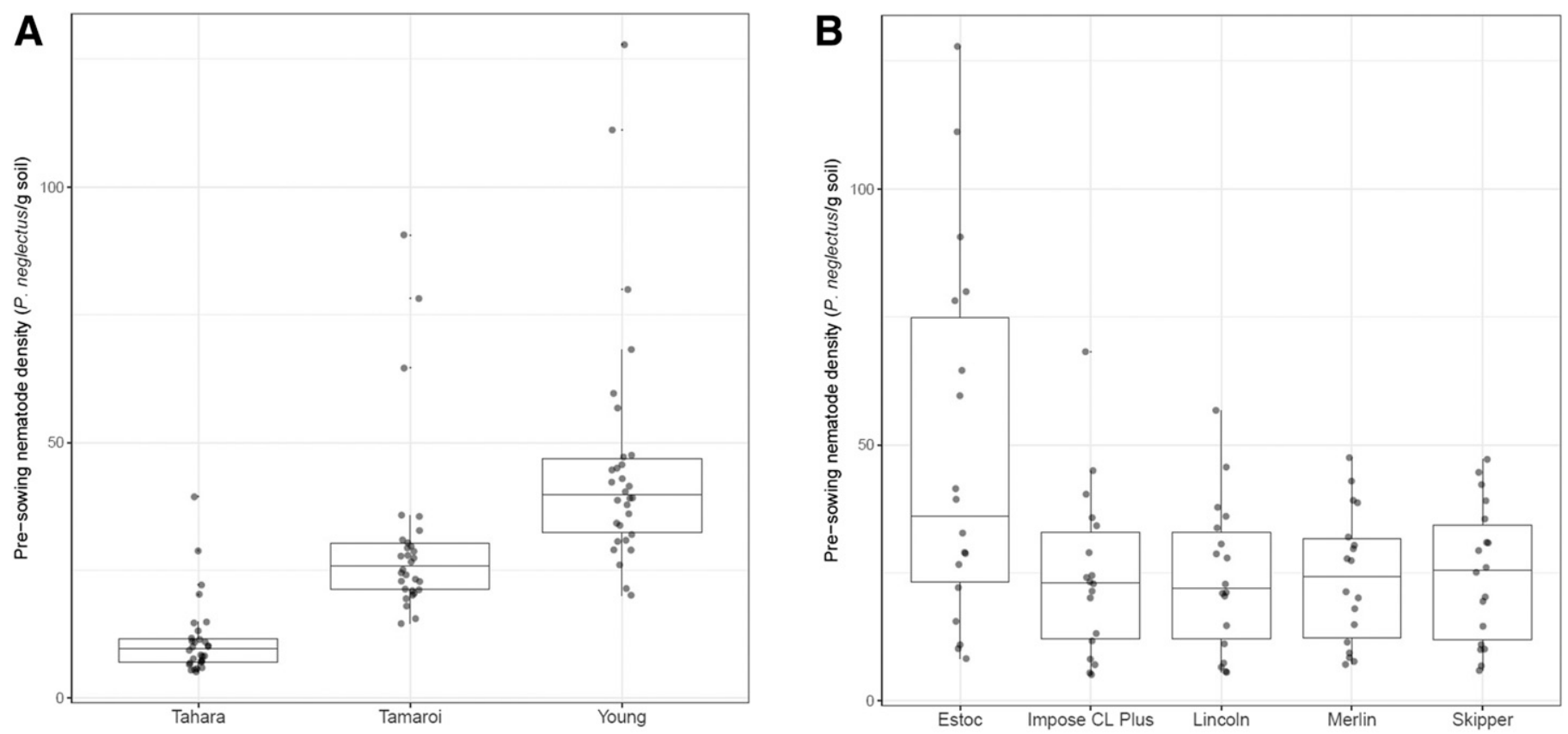

Fig. 1. Distributions of the Pratylenchus neglectus density levels prior to sowing in the second year of the Longerenong experiment grouped by cultivar sown in the A, set-up year and B, experimental year. Data points have been jittered for display purposes. The cultivar Estoc was randomized to all experiment plots with high presowing $P$. neglectus density levels by chance. 
ratios of variance components. Interaction terms were tested using REML ratio tests (Verbyla et al. 1999). Predicted means were derived for each cultivar at each observation point. Predicted means were then back-transformed and plotted in the original scale.

All analyses were implemented using the ASReml-R package (Butler et al. 2018) in the R statistical computing environment ( $R$ Core Team 2019), with variance components estimated using REML (Patterson and Thompson 1971).

\section{RESULTS}

Longerenong experiment. The ranges of $P i$ for the set-up cultivars were 5.1 to 39.4 P. neglectus $/ \mathrm{g}$ of soil for triticale cultivar Tahara, 14.6 to $90.6 \mathrm{P}$. neglectus/g of soil for durum cultivar Tamaroi, and 20.1 to 127.8 P. neglectus/g of soil for wheat cultivar
Young. The set-up cultivars did not create distinct levels of low, medium, and high RLN densities as intended, but instead induced overlapping populations that could be considered as a continuous scale, with considerable overlap between the higher levels induced by the durum cultivar Tamaroi and lower levels induced by the wheat cultivar Young (Fig. 1A). By allocating cultivars in the experimental year randomly to subplots within set-up cultivar main plots, cultivar Estoc was assigned to a far greater range of $P$. neglectus densities than the four other cultivars (Fig. 1B), with the difference in mean $P i$ density statistically significant $(\mathrm{F}(\mathrm{df}=4$, $80)=5.1, P=0.001)$. As the randomization for the experimental year was prepared during the set-up year, well in advance of soil sampling and without knowledge of the $P i$, the allocation of cultivar Estoc to all subplots with high outlying $P$. neglectus densities occurred by chance. The $P i$-conditioned randomization approach

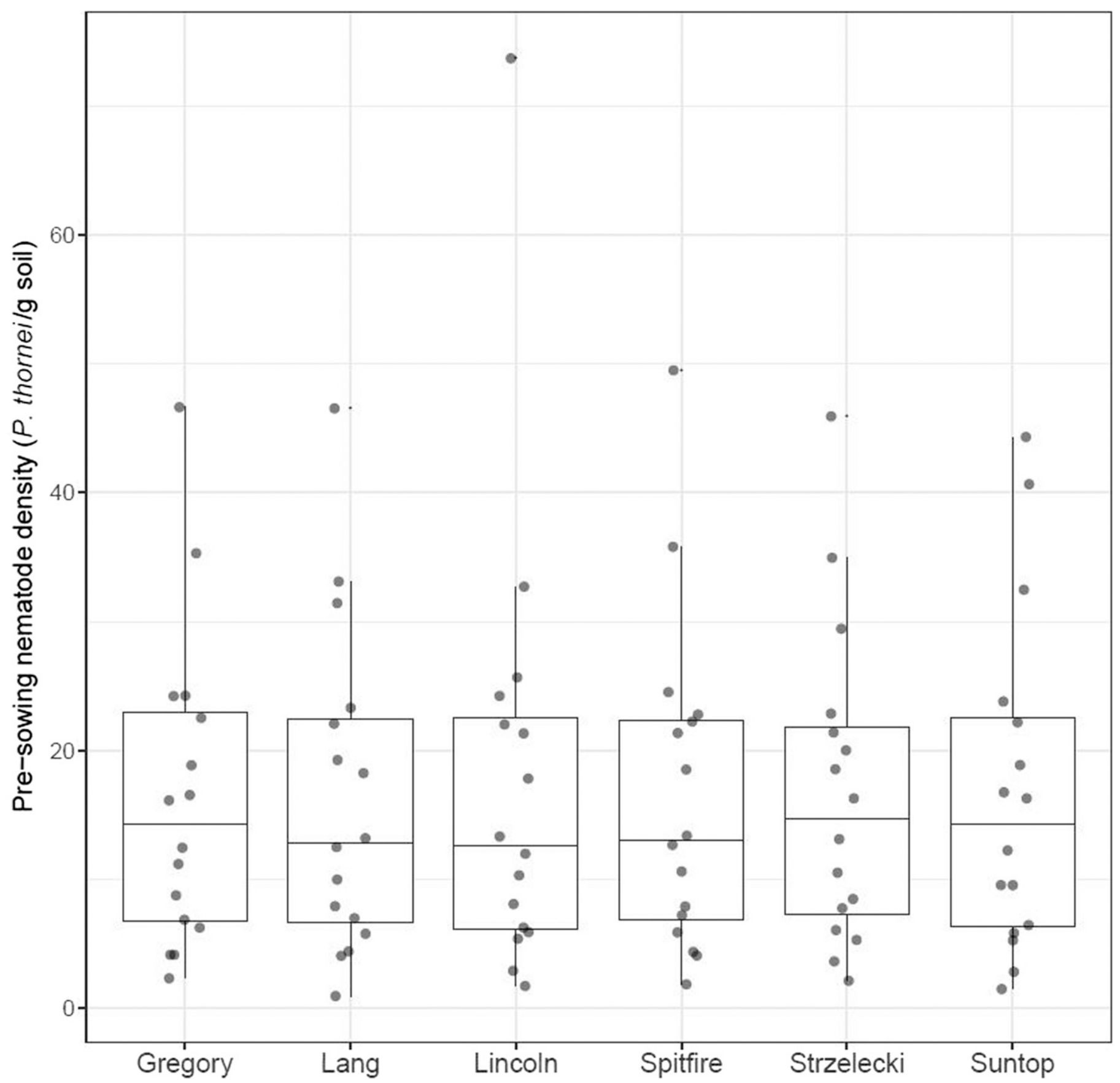

Fig. 2. Distributions of the Pratylenchus thornei density levels prior to sowing in the second year of the Formartin experiment grouped by cultivar sown in the experimental year. Data points have been jittered for display purposes. The alignment of quartiles demonstrates that the Pratylenchus sp. initial population density (measured prior to sowing)-conditioned randomization ensured cultivars were exposed to consistent ranges of $P$. thornei densities. 
was applied to the Longerenong $P i$ data and plot structure as an exercise, with one hundred alternative randomizations created (Table 1). A comparison of IQRs across cultivars shows that there was less variability exhibited with the constrained randomization simulated designs compared with the original randomization, with a maximum IQR of 26.7 across all simulations compared with 51.5 for the randomization used. By constraining the randomization of cultivars within blocks of $P i$ densities, the conditioned randomization experimental design approach consistently ensured that $P i$ density ranges were balanced across cultivars.

Formartin experiment. The range of $P i$ estimates was 1.0 to 73.7 P. thornei/g of soil, while the range of $P f$ estimates was 4.7 to 139.4 P. thornei/g of soil. The alignment of the box-plot medians and upper and lower quartiles in the plot of initial densities by experimental cultivar (Fig. 2) shows that the Pi-conditioned randomization approach ensured that each cultivar was exposed to approximately the same range of initial $P$. thornei densities, which allowed yield response curves to be compared. Curves modeling the relationship between pre- and postsowing nematode densities for each cultivar are provided in Figure 3. The interactions between cultivar and the spline term $\left(X^{2}(\mathrm{df}=1)=0.0, P=1\right)$ and between cultivar and presowing $P$. thornei density $\left(X^{2}(\mathrm{df}=1)=0.1, P=0.726\right)$ were not significant and were omitted from the final model. This indicated that cultivar slopes for square root-transformed $P f$ were parallel. The spline term was significant, indicating a nonlinear relationship between square root-transformed $P f$ and the presowing RLN density $\left(X^{2}(\mathrm{df}=1)=10, P=0.002\right)$. The gray dashed line in each panel of Figure 3 indicates the point of equivalence between $P i$ and $P f$ density of $P$. thornei. Estimated $P f$ was least for Suntop, and it was the only cultivar for which postharvest density was similar to or lower than presowing density across all levels of Pi. Other cultivars showed high reproduction, particularly at lower $P$. thornei levels. Population densities of $P$. thornei postharvest were greatest for the susceptible cultivars Lang, Lincoln and Strzelecki. There was an intermediate range of $P f$ for cultivars Gregory and Spitfire compared

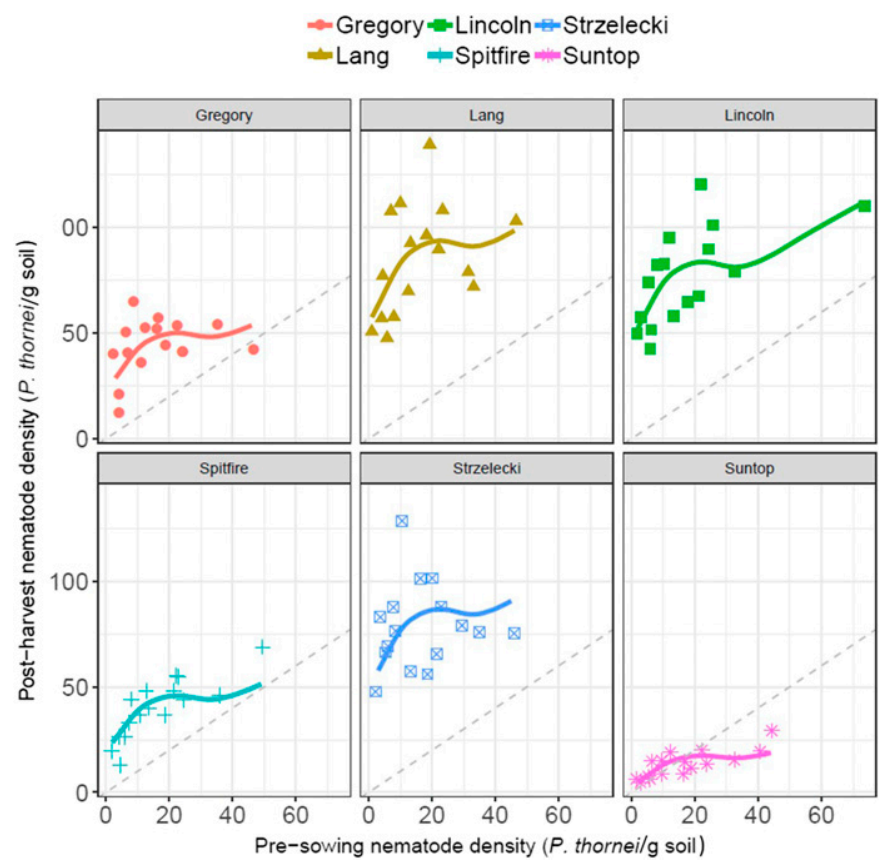

Fig. 3. Back-transformed curves describing the relationship between postharvest and presowing estimates of Pratylenchus thornei/g of soil for six wheat cultivars in the experiment conducted at Formartin. Symbols and lines correspond to different cultivars. The dashed line in each panel indicates the point of equivalence between Pratylenchus sp. initial population density measured prior to sowing and Pratylenchus sp. final population density measured after harvest. with cultivar Suntop, which was more resistant than cultivars Lang, Lincoln, and Strzelecki.

For the yield response curves, the interactions between cultivar and natural $\log$ transformed $P$. thornei density were significant in the models fitted for both initial $\left(X^{2}(\mathrm{df}=1)=11, P<0.001\right)$ and final $\left(X^{2}(\mathrm{df}=1)=5.2, P=0.022\right) P$. thornei densities, indicating that cultivar yield response curves were not parallel and the rates of yield loss varied between the cultivars. For the regression of yield against $\log$ transformed $P$. thornei densities measured prior to sowing, the slope estimates for cultivars Gregory, Spitfire, and Suntop were not significantly different from zero (Table 2), while for the regression of yield against $\log$ transformed $P$. thornei densities after harvest, all slope estimates were significantly different from zero (Table 3). Yield reduction from nematode infestation varied among the cultivars and was associated with nematode densities measured both presowing and postharvest (Figs. 4 and 5). Yield of all cultivars was reduced with increasing $P f$, with all slopes describing cultivar relationships between yield and $\log$ final $P$. thornei density significantly different from zero (Table 3). Yield reduction was least for cultivar Suntop and was associated with the most constrained $P f$ range (Fig. 5B). Yield losses for cultivars Gregory and Spitfire were comparable to that of cultivar Suntop but to a greater extent associated with higher $P f$ (and no plots had very low $P f$ ). Lang, Lincoln, and Strzelecki had the highest extent of yield loss associated with the highest levels of $P f$ (and no plots with low Pf [Figs. 4B and 5B]). Within the group of most susceptible varieties, the slope of Lang differed significantly from the slopes of Lincoln $(z=5.2, P<0.001$, two-tailed) and Strzelecki $(z=7.8, P<0.001$, two-tailed).

\section{DISCUSSION}

The experimental design proposed in this paper and implemented in the Formartin experiment incorporated a 2-year approach to ensure cultivars were exposed to consistent and wide ranges of presowing $P$. thornei population densities. The wide range of densities allowed the relationship between yield and RLN density to be modeled, and the consistency of the ranges of presowing $P$. thornei densities to which cultivars were exposed ensured comparisons between cultivars with respect to tolerance and

TABLE 2. Intercept and slope values for yield ( $\mathrm{t} / \mathrm{ha}$ ) response curves fitted for natural $\log$ transformed Pratylenchus sp. initial population density measured prior to sowing in the Formartin experiment ${ }^{\mathrm{a}}$

\begin{tabular}{lcc}
\hline Cultivar & Intercept \pm E.S.E. & Slope \pm E.S.E. \\
\hline Gregory & $4.252 \pm 0.188$ & $-0.063 \pm 0.056$ \\
Lang & $3.769 \pm 0.181$ & $-0.251 \pm 0.054$ \\
Lincoln & $3.379 \pm 0.181$ & $-0.410 \pm 0.054$ \\
Spitfire & $4.181 \pm 0.181$ & $-0.095 \pm 0.055$ \\
Strzelecki & $3.235 \pm 0.182$ & $-0.448 \pm 0.055$ \\
Suntop & $4.520 \pm 0.180$ & $-0.005 \pm 0.054$ \\
\hline
\end{tabular}

a E.S.E. gives the estimated standard error of the intercept and slope predictions.

TABLE 3. Intercept and slope values for yield ( $\mathrm{t} / \mathrm{ha}$ ) response curves fitted for natural $\log$ transformed Pratylenchus sp. final population density measured after harvest in the Formartin experiment ${ }^{\mathrm{a}}$

\begin{tabular}{lcc}
\hline Cultivar & Intercept \pm E.S.E. & Slope \pm E.S.E. \\
\hline Gregory & $5.257 \pm 0.380$ & $-0.312 \pm 0.098$ \\
Lang & $5.256 \pm 0.380$ & $-0.479 \pm 0.083$ \\
Lincoln & $5.256 \pm 0.380$ & $-0.672 \pm 0.085$ \\
Spitfire & $5.257 \pm 0.380$ & $-0.360 \pm 0.100$ \\
Strzelecki & $5.256 \pm 0.380$ & $-0.723 \pm 0.085$ \\
Suntop & $5.257 \pm 0.380$ & $-0.295 \pm 0.141$ \\
\hline
\end{tabular}

a E.S.E. gives the estimated standard error of the intercept and slope predictions. 
resistance were valid. Constraints were applied to the randomization to ensure that any potential for confounding with factors associated with the set-up cultivars was controlled through the design. This means that we can be confident that the yield responses are due to the nematode population densities and not to variation from other factors associated with the set-up conditions. Furthermore, the consistent ranges of initial nematode population densities among the experimental cultivars ensure that we can be confident that differences in nematode population densities observed after harvest arose from differences in the expression of resistance among the cultivars.
The approach of establishing initial nematode densities by implementing a randomization scheme for the experimental cultivars, which only randomizes within sets of plots sown to setup cultivars in the first year, cannot ensure that experimental cultivars will be exposed to consistent ranges of presowing nematode densities. This was demonstrated with the Longerenong experiment, in which cultivar Estoc was assigned to a far greater range of presowing $P$. neglectus densities than the other experimental cultivars by chance. Through the exercise of creating simulated rerandomizations for the experimental year of the Longerenong experiment using the proposed $P i$-conditioned

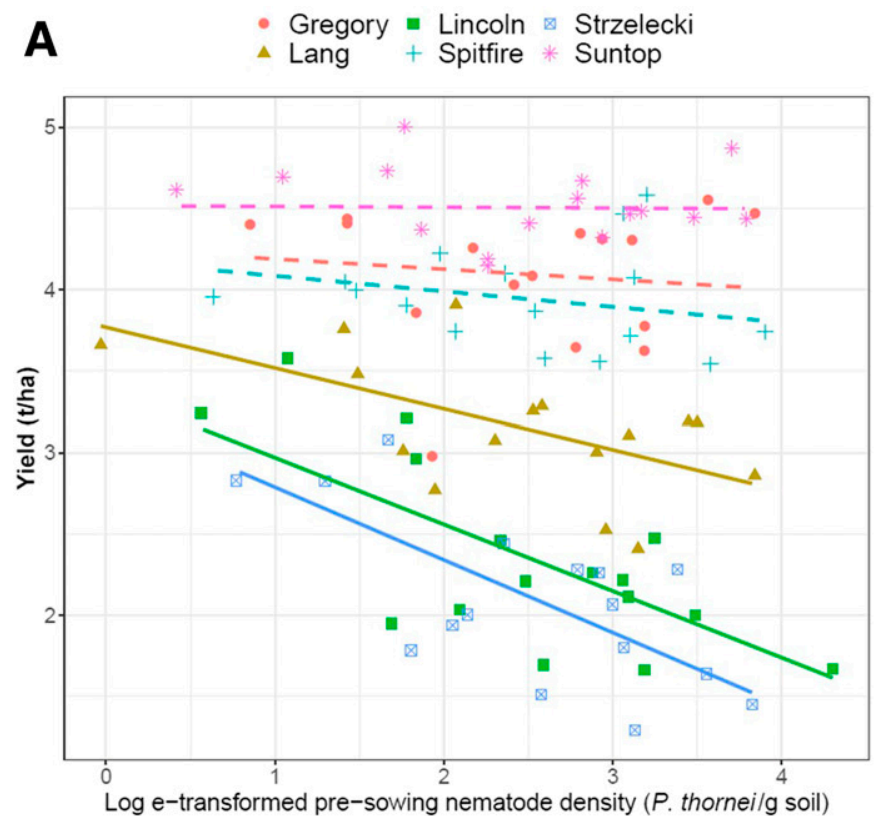

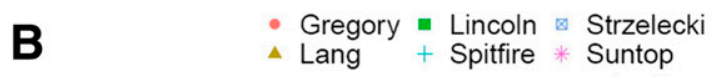

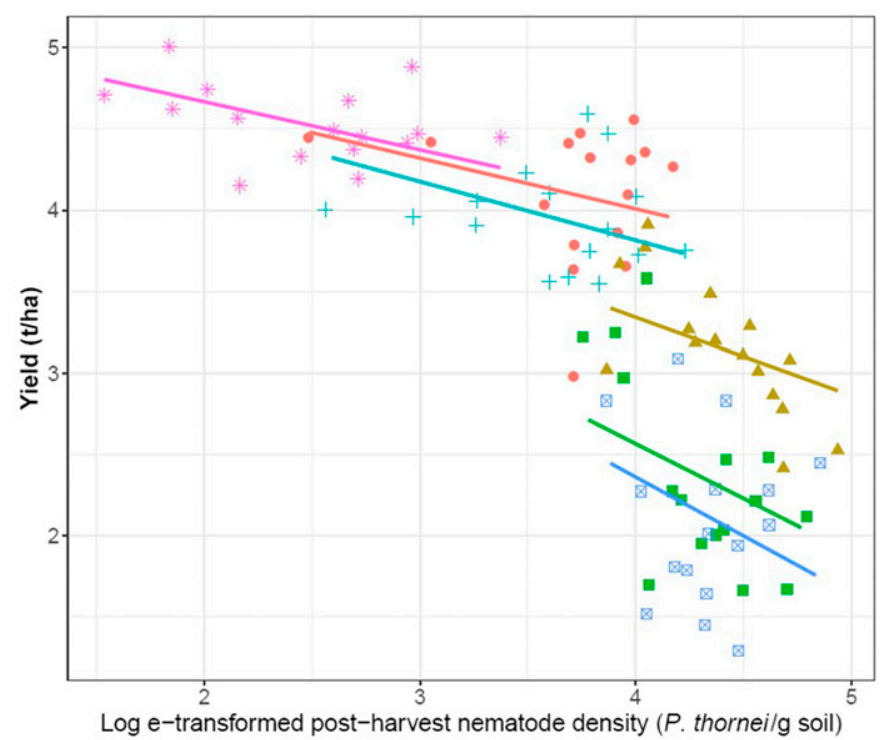

Fig. 4. Yield response curves describing the relationship between yield (t/ha) and $\mathbf{A}, \log$ initial and $\mathbf{B}, \log$ final Pratylenchus thornei/g of soil for six wheat cultivars in the experiment conducted at Formartin. Symbols and lines correspond to different cultivars. Dashed lines indicate slopes that are not significantly different from zero.
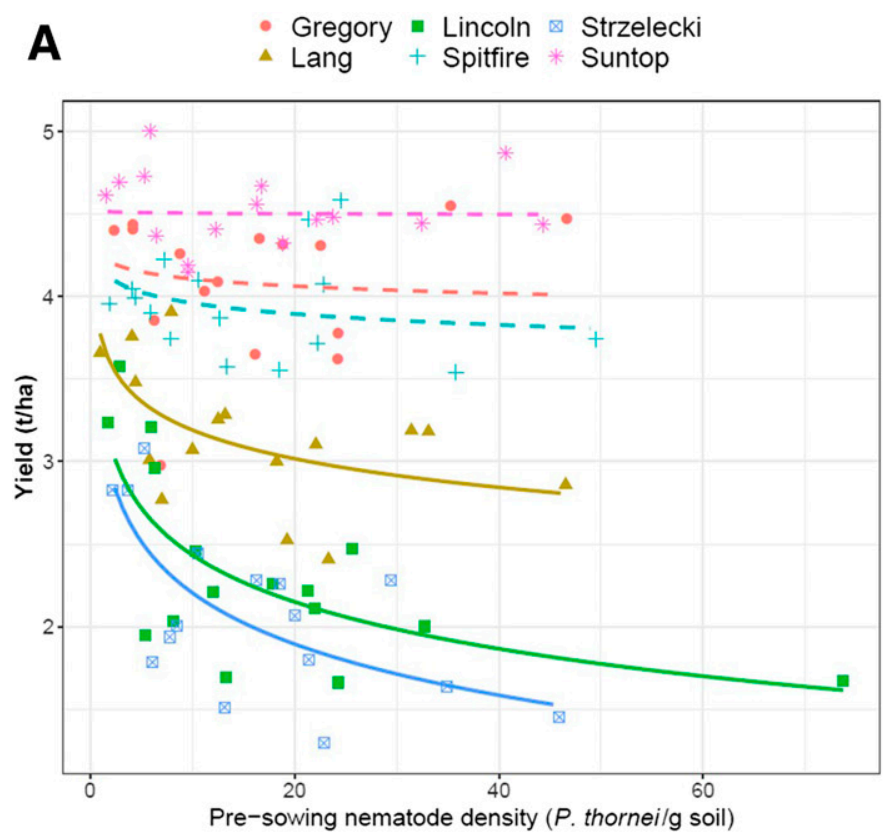
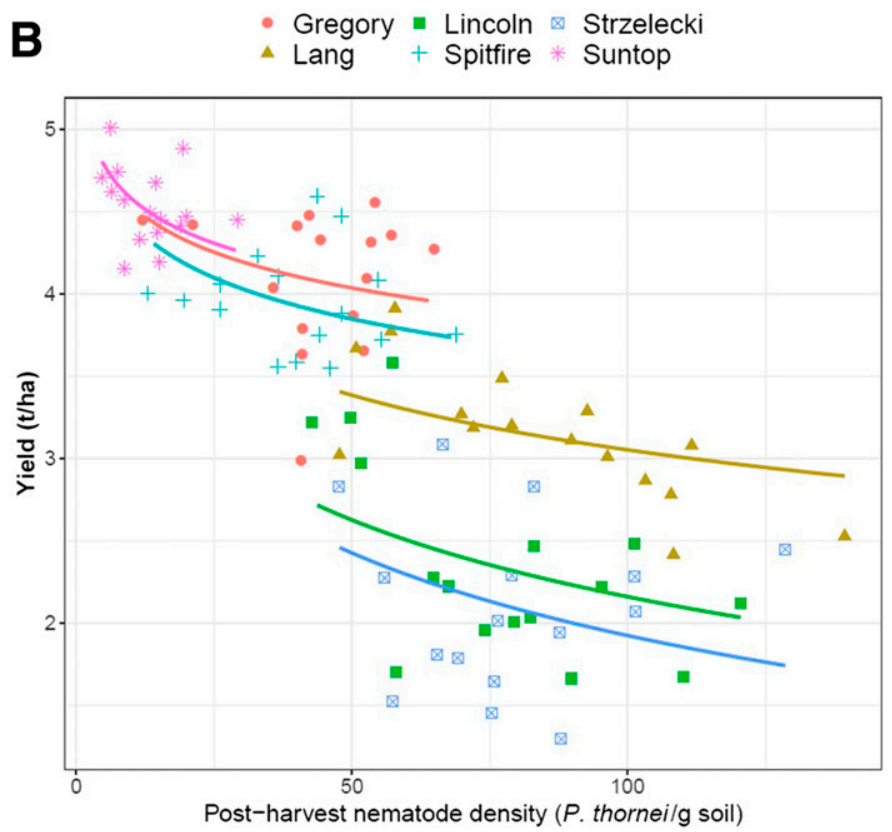

Fig. 5. Back-transformed yield response curves describing the relationship between yield and A, initial and B, final Pratylenchus thornei/g of soil for six wheat cultivars in the experiment conducted at Formartin. Symbols and lines correspond to different cultivars. Dashed lines indicate slopes that are not significantly different from zero. 
approach, we demonstrated that the new approach avoided this problem.

In order to fully assess the risk posed by RLNs to current and future crops, it is important to understand the relationship between increasing nematode burden and yield, together with the population dynamics of RLNs, and how these relationships vary between cultivars. Response curves modeling yield against $P i$ provided information on the risk of yield loss as a consequence of cultivar choice, which can be of benefit to a grower where a presowing diagnostic test (such as that offered by the PREDICTA B testing service) has been used to estimate the density of RLNs. As the Piconditioned experimental design approach ensures cultivars are exposed to consistent ranges of initial RLN population densities, response curves modeling yield against $P f$ can provide additional information on both yield loss as a function of experienced RLN burden and the population dynamics of RLN. Curves derived from a regression of yield against $P i$ showed that three of the six wheat cultivars in the Formartin experiment exhibited a significant negative rate of change in yield under increasing presowing $P$. thornei density, with the other three cultivars exhibiting a rate of yield loss not significantly different from zero. Yield regressed against $P f$ revealed that all cultivars lost yield associated with increasing $P f$. This information, however, only partially describes cultivar performance. The high degree to which final population densities were constrained for more resistant cultivars suggests that resistance was responsible for maintaining yield in a number of cultivars. Cultivars that expressed resistance to P. thornei not only had higher yields than highly susceptible cultivars, they also constrained the nematode population. This confers a benefit for susceptible crops grown in a subsequent season (Fanning et al. 2020; Owen et al. 2014).

The range of $P f$ values to which cultivars are exposed is dependent on an interplay between seasonal and environmental conditions and the resistance level of the cultivar. Factors such as temperature and soil moisture greatly influence hatching and penetration of susceptible host roots by Pratylenchus spp. (Castillo et al. 1996), and as a result, differences in both host response and nematode reproduction are observed between experiments, whether they are conducted under controlled conditions or in the field (De Waele and Elsen 2002). As the experimental design approach ensured that the cultivars were exposed to consistent ranges of presowing RLN densities, the regression of yield against $P f$ can illustrate both relative cultivar resistance and relative cultivar tolerance. The degree to which the cultivar-specific yield response curves extend along the $\mathrm{x}$-axis provides a measure of relative resistance. Three resistance levels were identified through a comparison of the relative $P f$ levels that developed in the Formartin experiment from the constrained $P i$ ranges: Suntop displayed the highest resistance with all estimated $P f$ below 30 P. thornei/g of soil, Gregory and Spitfire formed a less resistant grouping with all estimated $P f$ below 70 P. thornei/g of soil, while Lang, Lincoln, and Strzelecki formed a susceptible grouping with all estimated $P f$ below 140 P. thornei/g of soil.

Tolerance is described by the rate of change in yield as pathogen burden increases and is equivalent to the slope of a regression between yield and pathogen burden (Råberg et al. 2009). In general, the approach of Forknall et al. (2019) can distinguish the effects of resistance and tolerance. For this dataset, however, comparisons between cultivars from differing resistance classes would require an assumption of a consistent linear relationship beyond the observed range of $P f$ for the more resistant varieties. Differences in slope were observed between cultivars Lang and Strzelecki and cultivars Lang and Lincoln across equivalent final $P$. thornei population densities (i.e., equivalent susceptibility). This indication that Lang may be more tolerant than Strzelecki or Lincoln while being equally susceptible should be further investigated.

As nematode densities are assessed at both sowing and harvest in RLN experiments conducted in the field, curves can be fitted to model the relationship between final and initial nematode densities over a range of $P i$ levels. Alternative approaches to modeling this relationship include fitting a logarithmic relationship (Fanning et al. 2020) or using polynomial regression (Nicol et al. 1999). Rather than assuming and imposing a functional form on the curves, we describe an alternative approach that incorporated splines in the regression model and allowed the observed data to inform the curve shape. The curves modeling the change in P. thornei density were parallel with respect to square-root transformed $P i$ for all cultivars, with a higher rate of nematode multiplication at low presowing levels compared with higher presowing levels. The parallel lines do not imply identical levels of cultivar resistance, however, as the resistance level of the cultivar is reflected in the intercept. The dip in the curves for values of $P i>25$ may be data-driven or may reflect a reduction in nematode multiplication in heavily infested soils through a reduction in food sources (Ferris 1985). As noted above, Suntop was the most resistant, cultivars Gregory and Spitfire formed a less-resistant grouping, and cultivars Lang, Lincoln, and Strzelecki formed a susceptible grouping.

Conclusions. This paper presents an experimental design approach that helps ensure that cultivars under field evaluation for yield responses, as influenced by resistance or tolerance, are exposed to consistent ranges of presowing RLN densities. The benefits of the experimental design approach include the following: (i) avoidance of confounding effects from treatments used to induce variation in nematode density levels; (ii) ensuring the comparisons between cultivars are not biased; (iii) allowing a valid comparison of cultivar resistance by controlling the range of $P i$ fairly across cultivars; and (iv) enabling an assessment of tolerance by ensuring each cultivar is exposed to a wide and consistent range of initial RLN densities, and ensuring the influence of resistance is controlled in the assessment of yield loss. Implementing the design and analysis approach in trials conducted across different environments and in different seasons will be the subject of future research.

\section{ACKNOWLEDGMENTS}

We thank project DAW00245 module leaders Steven Simpfendorfer and Manisha Shankar for their contributions; Tim Clewett, Graham Exell, and Jordan McDonald for technical assistance; and grower Alex Gwynne and family for the use of their land.

\section{LITERATURE CITED}

Bailey, R. A. 2008. Design of Comparative Experiments. Cambridge University Press, Cambridge, U.K.

Barker, K. R., Koenning, S. R., Bostian, A. L., and Ayers, A. R. 1988. Growth and yield responses of soybean to Aldicarb. J. Nematol. 20:421-431.

Barker, K. R., and Olthof, T. H. A. 1976. Relationships between nematode population densities and crop responses. Annu. Rev. Phytopathol. 14: 327-353.

Butler, D. G. 2013. On the optimal design of experiments under the linear mixed model. Ph.D. thesis, School of Mathematics and Physics, The University of Queensland, Australia.

Butler, D. G., Cullis, B. R., Gilmour, A. R., and Gogel, B. J. 2018. ASReml-R 4 reference manual: Mixed models for S language environments. Queensland Department of Primary Industries and Fisheries.

Castillo, P., Trapero-Casas, J. L., and Jimenez-Diaz, R. M. 1996. The effect of temperature on hatching and penetration of chickpea roots by Pratylenchus thornei. Plant Pathol. 45:310-315.

De Waele, D., and Elsen, A. 2002. Migratory endoparasites: Pratylenchus and Radopholus species. Pages 175-206 in: Plant Resistance to Parasitic Nematodes. J. R. Starr, R. Cook, and J. Bridge, eds. CABI, Wallingford, U.K.

Elston, D. A., Phillips, M. S., and Trudgill, D. L. 1991. The relationship between initial population density of potato cyst nematode Globodera pallida and the yield of partially resistant potatoes. Rev. Nematol. 14: 221-229.

Fanning, J., Linsell, K., McKay, A., Gogel, B., Munoz Santa, I., Davey, R., and Hollaway, G. 2018. Resistance to the root lesion nematodes Pratylenchus thornei and P. neglectus in cereals: Improved assessments in the field. Appl. Soil Ecol. 132:146-154. 
Fanning, J., Reeves, K., Forknall, C., McKay, A., and Hollaway, G. J. 2020. Pratylenchus thornei: The relationship between pre-sowing nematode density and yield loss in wheat and barley. Phytopathology 110:674-683.

Ferris, H. 1985. Density-dependent nematode seasonal multiplication rates and overwinter survivorship: A critical point model. J. Nematol. 17:93-99.

Fisher, R. A. 1935. The Design of Experiments. Oliver \& Boyd, Oxford, England.

Forknall, C. R., Simpfendorfer, S., and Kelly, A. M. 2019. Using yield response curves to measure variation in the tolerance and resistance of wheat cultivars to Fusarium crown rot. Phytopathol. 109:932-941.

Gilmour, A. R., Cullis, B. R., and Verbyla, A. P. 1997. Accounting for natural and extraneous variation in the analysis of field experiments. JABES 2:269-293.

Gomez, K. A., and Gomez, A. A. 1984. Statistical Procedures for Agricultural Research, 2nd ed. John Wiley \& Sons, New York.

Hollaway, G. J., Ophel-Keller, K. M., Taylor, S. P., Burns, R. A., and McKay, A. C. 2003. Effect of soil water content, sampling method and sample storage on the quantification of root lesion nematodes (Pratylenchus spp.) by different methods. Australas. Plant Pathol. 32:73-79.

Isbell, R. F. 1996. The Australian Soil Classification. CSIRO Publishing, Melbourne.

Kagoda, F., Derera, J., Tongoona, P., Coyne, D. L., and Talwana, H. L. 2011. Grain yield and heterosis of maize hybrids under nematode infested and nematicide treated conditions. J. Nematol. 43:209-219.

Kause, A., and Odegard, J. 2012. The genetic analysis of tolerance to infections: A review. Front. Genet. 3:262.

Kelly, A. M., and Forknall, C. R. 2020. Advanced designs for barley breeding experiments. Achieving Sustainable Cultivation of Barley. G. P. Fox and C. Li, eds. Burleigh Dodds Science Publishing Limited, Cambridge, U.K.

Linsell, K. J., Davey, R. S., Fanning, J., Hollaway, G., Munoz Santa, S., Gogel, B., and McKay, A. 2015. Assessing resistance and tolerance of cereal varieties to root lesion nematodes in multi-environment field trials. Pages 14-16 in: Australasian Plant Pathology Society Conference Proceedings. Fremantle, Western Australia.

Murray, G. M., and Brennan, J. P. 2009. Estimating disease losses to the Australian wheat industry. Australas. Plant Pathol. 38:558-570.

Nicol, J. M., Davies, K. A., Hancock, T. W., and Fisher, J. M. 1999. Yield loss caused by Pratylenchus thornei on wheat in South Australia. J. Nematol. 31:367-376.

Ophel-Keller, K., McKay, A., Hartley, D., Herdina, and Curran, J. 2008. Development of a routine DNA-based testing service for soilborne diseases in Australia. Australas. Plant Pathol. 37:243-253.

Owen, K. J., Clewett, T. G., Bell, K. L., and Thompson, J. P. 2014. Wheat biomass and yield increased when populations of the root lesion nematode (Pratylenchus thornei) were reduced through sequential rotation of partially resistant winter and summer crops. Crop Pasture Sci. 65:227-241.

Owen, K. J., Clewett, T. G., and Thompson, J. P. 2010. Pre-cropping with canola decreased Pratylenchus thornei populations, arbuscular mycorrhizal fungi, and yield of wheat. Crop Pasture Sci. 61:399-410.

Patterson, H. D., and Thompson, R. 1971. Recovery of inter-block information when block sizes are unequal. Biometrika 58:545-554

R Core Team. 2019. R: A language and environment for statistical computing. R Foundation for Statistical Computing, Vienna, Austria.
Råberg, L., Graham, A. L., and Read, A. F. 2009. Decomposing health: Tolerance and resistance to parasites in animals. Philos. Trans. R. Soc. Lond. B Biol. Sci. 364:37-49.

Reen, R. A., Thompson, J. P., Clewett, T. G., Sheedy, J. G., and Bell, K. L. 2014. Yield response in chickpea cultivars and wheat following crop rotations affecting population densities of Pratylenchus thornei and arbuscular mycorrhizal fungi. Crop Pasture Sci. 65:428-441.

Roberts, P. A. 2002. Concepts and consequences of resistance. Pages 23-41 in: Plant Resistance to Parasitic Nematodes. J. R. Starr, R. Cook, and J. Bridge, eds. CABI, Wallingford, U.K.

Sheedy, J. G., McKay, A. C., Lewis, J., Vanstone, V. A., Fletcher, S., Kelly, A., and Thompson, J. P. 2015. Cereal cultivars can be ranked consistently for resistance to root-lesion nematodes (Pratylenchus thornei \& P. neglectus) using diverse procedures. Australas. Plant Pathol. 44:175-182.

Sheedy, J. G., and Thompson, J. P. 2009. Resistance to the root-lesion nematode Pratylenchus thornei of Iranian landrace wheat. Australas. Plant Pathol. 38:478-489.

Smiley, R. W., Whittaker, R. G., Gourlie, J. A., and Easley, S. A. 2005. Pratylenchus thornei associated with reduced wheat yield in Oregon. J. Nematol. 37:45-54.

Stefanova, K. T., Smith, A. B., and Cullis, B. R. 2009. Enhanced diagnostics for the spatial analysis of field trials. JABES 14:392-410.

Taylor, S. P., Vanstone, V. A., Ware, A. H., McKay, A. C., Szot, D., and Russ, M. H. 1999. Measuring yield loss in cereals caused by root lesion nematodes (Pratylenchus neglectus and P. thornei) with and without nematicides. Aust. J. Agric. Res. 50:617-622.

Thompson, J. P., Brennan, P. S., Clewett, T. G., Sheedy, J. G., and Seymour, N. P. 1999. Progress in breeding wheat for tolerance and resistance to root-lesion nematode (Pratylenchus thornei). Australas. Plant Pathol. 28:45-52.

Thompson, J. P., Mackenzie, J., and Clewett, T. G. 1989. Rapid coring of clay topsoils for microbiological analysis. Australas. Plant Pathol. 18:1-2.

Thompson, J. P., Mackenzie, J., and Sheedy, G. H. 2012. Root-lesion nematode (Pratylenchus thornei) reduces nutrient response, biomass and yield of wheat in sorghum-fallow-wheat cropping systems in a subtropical environment. Field Crops Res. 137:126-140.

Trudgill, D. L. 1991. Resistance to and tolerance of plant parasitic nematodes in plants. Annu. Rev. Phytopathol. 29:167-192.

Vanstone, V. A., Hollaway, G. J., and Stirling, G. R. 2008. Managing nematode pests in the southern and western regions of the Australian cereal industry: Continuing progress in a challenging environment. Australas. Plant Pathol. 37:220-234

Vanstone, V. A., Rathjen, A. J., Ware, A. H., and Wheeler, R. D. 1998. Relationship between root lesion nematodes (Pratylenchus neglectus and P. thornei) and performance of wheat varieties. Aust. J. Exp. Agric. 38: 181-188.

Verbyla, A. P., Cullis, B. R., Kenward, M. G., and Welham, S. J. 1999. The analysis of designed experiments and longitudinal data by using smoothing splines. J. R. Stat. Soc. C-Appl. 48:269-311.

Whish, J. P. M., Thompson, J. P., Clewett, T. G., Lawrence, J. L., and Wood, J. 2014. Pratylenchus thornei populations reduce water uptake in intolerant wheat cultivars. Field Crops Res. 161:1-10. 